\title{
An experimental and theoretical study of nitrogen-broadened acetylene lines
}

\author{
Franck Thibault ${ }^{\mathrm{a}, *}$, Raúl Z. Martínez ${ }^{\mathrm{b}}$, Dionisio Bermejo ${ }^{\mathrm{b}}$, Sergey V. Ivanov ${ }^{\mathrm{c}}$, \\ Oleg G. Buzykin ${ }^{\mathrm{d}}$, Qiancheng Ma ${ }^{\mathrm{e}}$ \\ a Institut de Physique de Rennes, UMR CNRS 6251, Université de Rennes I, Campus de Beaulieu, Bât. 11B, F-35042 Rennes, France \\ b Instituto de Estructura de la Materia, IEM-CSIC, Serrano 123, 28006 Madrid, Spain \\ ${ }^{c}$ Institute on Laser and Information Technologies, Russian Academy of Sciences, 2 Pionerskaya Str., 142190 Moscow, Troitsk, Russia \\ ${ }^{d}$ Central Aerohydrodynamic Institute (TSAGI), 140160 Zhukovski, Moscow Region, Russia \\ e NASA/Goddard Institute for Space Studies and Department of Applied Physics and Applied Mathematics, Columbia University, \\ 2880 Broadway, New York, NY 10025, USA
}

\section{A R T I C L E I N F O}

\section{Article history:}

Received 7 January 2014

Received in revised form

10 March 2014

Accepted 12 March 2014

Available online 21 March 2014

Keywords:

Ethyne

Acetylene

Nitrogen

Pressure broadening coefficients

Stimulated Raman spectroscopy

Dynamical methods

\begin{abstract}
A B S T R A C T
We present experimental nitrogen-broadening coefficients derived from Voigt profiles of isotropic Raman Q-lines measured in the $\nu_{2}$ band of acetylene $\left(\mathrm{C}_{2} \mathrm{H}_{2}\right)$ at $150 \mathrm{~K}$ and $298 \mathrm{~K}$, and compare them to theoretical values obtained through calculations that were carried out specifically for this work. Namely, full classical calculations based on Gordon's approach, two kinds of semi-classical calculations based on Robert-Bonamy method as well as full quantum dynamical calculations were performed. All the computations employed exactly the same ab initio potential energy surface for the $\mathrm{C}_{2} \mathrm{H}_{2}-\mathrm{N}_{2}$ system which is, to our knowledge, the most realistic, accurate and up-to-date one. The resulting calculated collisional half-widths are in good agreement with the experimental ones only for the full classical and quantum dynamical methods. In addition, we have performed similar calculations for IR absorption lines and compared the results to bibliographic values. Results obtained with the full classical method are again in good agreement with the available room temperature experimental data. The quantum dynamical closecoupling calculations are too time consuming to provide a complete set of values and therefore have been performed only for the $\mathrm{R}(0)$ line of $\mathrm{C}_{2} \mathrm{H}_{2}$. The broadening coefficient obtained for this line at $173 \mathrm{~K}$ and $297 \mathrm{~K}$ also compares quite well with the available experimental data. The traditional Robert-Bonamy semi-classical formalism, however, strongly overestimates the values of half-width for both Q- and R-lines. The refined semiclassical Robert-Bonamy method, first proposed for the calculations of pressure broadening coefficients of isotropic Raman lines, is also used for IR lines. By using this improved model that takes into account effects from line coupling, the calculated semi-classical widths are significantly reduced and closer to the measured ones.
\end{abstract}

(c) 2014 Elsevier Ltd. All rights reserved.

\footnotetext{
*Corresponding author. Tel.: +33223236182; fax: 33223235662.

E-mail address: Franck.Thibault@univ-rennes1.fr (F. Thibault).
}

\section{Introduction}

Many experimental infrared studies have been devoted to the nitrogen broadening of acetylene lines of various fundamentals or combination bands [1-9]. Table 1 of the recent studies of Predoi-Cross and collaborators [8,9] gives an exhaustive summary. In the present work, we complete 
Table 1

Pressure broadening coefficients for the $\mathrm{R}(0)$ line at room temperature $(297 \pm 2 \mathrm{~K}$ ), $173 \mathrm{~K}$ and $100 \mathrm{~K}$ (as a reference for future use, for instance, for Titan's atmosphere).

\begin{tabular}{llll}
\hline & Room T & $173 \mathrm{~K}$ & $100 \mathrm{~K}$ \\
\hline Calculated & $120.45(\mathrm{a})$ & $175.75(\mathrm{a})$ & $259.05(\mathrm{a})$ \\
& $119.75(\mathrm{~b})$ & $177.95(\mathrm{~b})$ & $264.85(\mathrm{~b})$ \\
& $121.05(\mathrm{c})$ & $176.30(\mathrm{c})$ & $258.50(\mathrm{c})$ \\
& $121.30(\mathrm{~d})$ & $177.95(\mathrm{~d})$ & $264.85(\mathrm{~d})$ \\
Experimental & $116.60(\mathrm{e})$ & $161.10(\mathrm{i})$ & \\
& $119.95(\mathrm{f})$ & $168.00(\mathrm{j})$ & \\
& $116.15(\mathrm{~g})$ & & \\
\hline & $110.00(\mathrm{~h})$ & & \\
\hline
\end{tabular}

(a) CC ThAv, (b) CC MThV, (c) CC/CS ThAv, (d) CC/CS MThV, (e) $\nu_{5}$ band [1] $297 \mathrm{~K}$, (f) $\nu_{1}+\nu_{3}$ band [5] $295 \mathrm{~K}$, (g) $\nu_{1}+\nu_{5}$ band $297 \mathrm{~K} \mathrm{[2],} \mathrm{(h)} \nu_{1}+\nu_{3}$ band [8], (i) $\nu_{4}+\nu_{5}$ band [6] $173.2 \mathrm{~K},(\mathrm{j}) \nu_{5}$ band $173.4 \mathrm{~K}[3]$.

these studies by performing the very first broadening measurement of isotropic Raman Q-branch lines in the $\nu_{2}$ band at $150 \mathrm{~K}$ and $298 \mathrm{~K}$. The motivation of this and similar works are well known, with the data obtained being of interest to (and finding application in) fields as diverse as the study of planetary atmospheres -including the Earth - combustion processes or pollution control.

Due to the interest of the $\mathrm{C}_{2} \mathrm{H}_{2}-\mathrm{N}_{2}$ system many theoretical calculations of the nitrogen broadening of acetylene lines have also been performed [1,3,4,8-11]. However, these calculations were almost all conducted with the semi-classical method of Robert and Bonamy [12] using semi-empirical potentials. For instance, in Ref. [8] various isotropic parts of this system were tested in order to get a good agreement with the experimental data. A few calculations were also performed on the basis of the energy corrected sudden approximation, for which no potential energy surface was used and for which the nitrogen molecule was reduced to a pseudo-atom $[13,14]$. The very first realistic dynamical calculations performed on an $a b$ initio potential energy surface (PES) [15] were done in Ref. [9] using the full classical method of Gordon $[16,17]$. In the present study, starting with the same PES [15], we are extending this effort. We intercompare the full classical method (FC) with semi-classical (SC) methods [12,18-20], quantum dynamical calculations, available experimental results for infrared lines and our results for isotropic Raman lines. In addition, this study completes a series of papers [21-23] devoted to a comparison of the full classical, the close-coupling (CC) and the RobertBonamy (RB) methods. In that series it has been shown that the traditional $\mathrm{RB}$ formalism is unable to provide accurate pressure broadening coefficients without adjusting some intermolecular parameters. More recently, Ma et al. [20] have shown that by considering effects from the line coupling in the standard [12] RB method, one is able to obtain a much better agreement with experimental values at least for self-perturbed nitrogen isotropic Raman lines. The latter study has been completed by a comparison of the full relaxation matrix as obtained with this refined method with quantum dynamical calculations $[24,25]$. Meanwhile, this refined version of semi-classical RB model has been adapted to the case of IR lines [26].
The aim of such comparative studies, including full classical ones, is to find well founded alternative methods to the quantum dynamical calculations, in particular to the CC method, which still remain very time consuming even for simple systems like $\mathrm{N}_{2}-\mathrm{N}_{2}$ or $\mathrm{C}_{2} \mathrm{H}_{2}-\mathrm{N}_{2}$.

In the following sections we briefly recall a few points about the methods used. Then the experimental set up and reduction of data are given. The results of our calculations are compared with our experimental Raman data and, finally, with bibliographic infrared data.

\section{Theoretical methods}

Binary collisions and the impact approximation for lines without line mixing [27] are the shared features of the methods summarized below. We disregard any translational effects (velocity or speed dependence) on the lineshape. Since the PES [15] is only four dimensional in the Jacobi coordinates, our calculations are performed within the rigid rotor approximation. We are therefore unable to provide any lineshift coefficients as well as to discuss any vibrational dependence of the collisional linewidths. The latter seems to be quite insensitive to the different kinds of transitions involving different vibrational modes [7] but the situation is not so clear regarding, for instance, Fig. 6 of [6], Fig. 13 of [7], Fig. 5 of [8] and Fig.4 of [9]. However, such experimental differences may arise because of the different lineshape models used. Regardless, our aim is not to provide accurate pressure broadening coefficients but to compare the methods we have used starting with the same PES.

\subsection{Potential energy surface used}

The calculations have been performed on the fourdimensional $a b$ initio PES of Thibault et al. [15]. This PES has been expanded over bispherical harmonics (see Refs. therein [23]):

$V\left(R, \theta_{1}, \theta_{2}, \Phi\right)=\sum_{L_{1}, L_{2}, L} V_{L_{1} L_{2} L}(R) \times A_{L_{1} L_{2} L}\left(\theta_{1}, \theta_{2}, \Phi\right)$

where $\left(\theta_{1}, \theta_{2}, \Phi\right)$ are the three angular Jacobi coordinates which describe the relative orientation of the two monomers. $R$ is the distance between the two monomer centers of mass and defines the intermolecular $z$ axis, $\theta_{\{1,2\}}$ defines the bending angle of the monomer axis relative to the intermolecular axis (the indices " 1 " and "2" refer to the acetylene and nitrogen molecules respectively) and $\Phi$ is the rotational angle around the collisional $\mathrm{z}$ axis oriented from " 1 " to " 2 ". The values $L_{1}, L_{2}$ and $L$ are even because the colliding pair is formed of centrosymmetric molecules and $\left|L_{1}-L_{2}\right| \leq L \leq\left|L_{1}+L_{2}\right|$. In Eq. (1) 85 terms have been used, up to $\left(L_{1}, L_{2}, L\right)=(8,8,16)$. The angular functions $A_{L_{1} L_{2} L}\left(\theta_{1}, \theta_{2}, \Phi\right)$ are defined in the case of two linear molecules by a normalized product of spherical harmonics for monomers 1 and 2 as

$$
\begin{aligned}
& A_{L_{1} L_{2} L}\left(\theta_{1}, \theta_{2}, \Phi\right) \\
& \quad=\left(\frac{2 L+1}{4 \pi}\right)^{1 / 2} \sum_{m}\left\langle L_{1} m L_{2}-m \mid L 0\right\rangle Y_{L_{1}}^{m}\left(\theta_{1}, 0\right) Y_{L_{2}}^{-m}\left(\theta_{2}, \Phi\right)
\end{aligned}
$$

where $Y_{L_{1}}^{m}$ and $Y_{L_{2}}^{-m}$ are the ordinary spherical harmonics, $\langle\ldots . . .$.$\rangle is a Clebsch-Gordan coefficient, and |m| \leq \min \left(L_{1}, L_{2}\right)$. 
The radial coefficients $V_{L_{1} L_{2} L}(R)$ were extrapolated, using $C_{\alpha} / R^{\alpha}$ functions, from 16 to $30 \AA$ since the $a b$ initio points end at $16 \AA$.

Such an expansion of the PES is suitable for the MOLSCAT quantum dynamical code [28] and for the classical calculations. However, in order to be used in conjunction with the RB method, in a fixed reference frame (Fig. 5 of [12]), a transformation has to be performed, leading to

$U_{L_{1}, L_{2}, M}(R)=\frac{1}{(4 \pi)^{3 / 2}} \sum_{L}(2 L+1)\left(\begin{array}{ccc}L_{1} & L_{2} & L \\ M & -M & 0\end{array}\right) V_{L_{1}, L_{2}, L}(R)$.

\subsection{Pressure broadening cross-sections and coefficients}

Considering $\mathrm{C}_{2} \mathrm{H}_{2}$ (index "1") in mixtures with natural $\mathrm{N}_{2}$ (index "2") composed of a 2:1 ratio of ortho- $\left(\mathrm{oN}_{2}\right)$ and para- $\left(\mathrm{pN}_{2}\right)$ species associated with even and odd rotational quantum number $j_{2}$, the broadening coefficient $\gamma\left(j_{1}, T\right)$ (in $\mathrm{cm}^{-1} \mathrm{~atm}^{-1}$ ) at a given temperature $T$ can be expressed as weighted sums of partial pressure broadening coefficients $[22,23,29,30]$ :

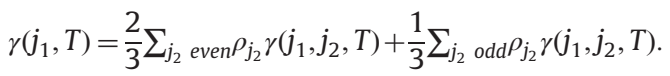
and

In Eq. (4) $\rho_{j_{2}}$ is the normalized rotational population

$\gamma\left(j_{1}, j_{2}, T\right)=f\left\langle v \sigma^{(n)}\left(j_{1}, j_{2}, E_{k i n}\right)\right\rangle \equiv f \bar{v} \sigma^{(n)}\left(j_{1}, j_{2}, T\right)$,

where $\sigma^{(n)}\left(j_{1}, j_{2}, T\right)$ is the Boltzmann average [29] over kinetic energy of the partial pressure broadening crosssections $\sigma^{(n)}\left(j_{1}, j_{2}, E_{k i n}\right)$ (in $\AA^{2}$ ) and $f=(D / 2 \pi c)(273 / T)$, where $D$ is the number density at standard temperature and pressure $\left(D=2.6910^{19}\right.$ molecule $\left.\mathrm{cm}^{-3}\right)$ and $c$ is the speed of light (in $\mathrm{cm} \mathrm{s}^{-1}$ ). $\bar{v}$ is the mean relative speed associated to the thermal speed distribution: $\bar{v}=\sqrt{8 \mathrm{k}_{B} T / \pi \mu}$, where $\mu$ is the reduced mass of the colliding pair in u units. Finally, the "exponent" $(n)$ denotes the order of the tensor of the interaction between matter and light ( $n=0$ for isotropic Raman lines and 1 for IR transitions).

An approximation often employed for the semi-classical method (for technical reasons) but also for CC (due to the prohibitive cost of such calculations) as well as for the fullclassical methods consists of providing pressure broadening coefficients without performing the thermal average over the distribution of relative kinetic energy of collisions. Within this approximation we consider that cross-sections are independent of translational energy and approximate the partial pressure broadening coefficient $\gamma\left(j_{1}, j_{2}, T\right)$ by its value at the mean relative speed $\bar{v}$, leading to [30]:

$\gamma\left(j_{1}, j_{2}, T\right)=f \bar{v} \sigma^{(n)}\left(j_{1}, j_{2} ; \bar{E}_{k i n}\right)$

with $\bar{E}_{\text {kin }}$ the kinetic energy associated with $\bar{v}$. Omitting the thermal average, Eq. (4) now reads

$$
\begin{aligned}
& \gamma\left(j_{A}, T\right)=f \bar{v}\left[\frac{2}{3} \sum_{j_{B} \text { even }} \rho_{j_{B}} \sigma^{(n)}\left(j_{A}, j_{B}, \bar{E}_{k i n}\right)\right. \\
& \left.+\frac{1}{3} \sum_{j_{B} \text { odd }} \rho_{j_{B}} \sigma^{(n)}\left(j_{A}, j_{B}, \bar{E}_{k i n}\right)\right] .
\end{aligned}
$$

In the following paragraphs, calculated values obtained through the use of Eq. (5) will be denoted as ThAv, while values obtained with Eq. (7) will be denoted as MThV for mean thermal velocity.

\subsection{Quantum dynamical methods}

Both the close-coupling [31-33] and coupled-states (centrifugal sudden approximation) [34] methods were used. The Appendix A provides the link between the diffusion scattering matrix and the pressure broadening cross-sections. We also refer the reader to Ref. [23] for technical details about dynamical calculations which are very similar to the one used in the present study.

Because of the relatively large reduced mass of the $\mathrm{C}_{2} \mathrm{H}_{2}-\mathrm{N}_{2}$ system, the small rotational constants $\left(B_{1}=\right.$ $\left.1.1766 \mathrm{~cm}^{-1}, B_{2}=1.998 \mathrm{~cm}^{-1}\right)$, in particular that of acetylene, and the large number of terms of the PES, quantum dynamical calculations turned out to be very time consuming ${ }^{1}$ and demanded a large amount of memory. Consequently, we were unable to perform CC calculations for total energies $\left(E_{\text {kin }}+E_{\text {rot }}\left(j_{1}\right)+E_{\text {rot }}\left(j_{2}\right)\right)$ greater than $350 \mathrm{~cm}^{-1}$, and CS calculations were performed up to only $700 \mathrm{~cm}^{-1}$. It is worth comparing this situation with our recent studies of $\mathrm{C}_{2} \mathrm{H}_{2}-\mathrm{H}_{2}$ [21] and $\mathrm{N}_{2}-\mathrm{H}_{2}$ [22], where the same problems of computational capacity were present but -due to the nature of these systems - to a lesser extent and higher energies could be reached. Also, simple extrapolation functions were used in these works when even higher energies needed to be accessed.

In order to compute the pressure broadening coefficients of the $\mathrm{R}(0)$ line we have only computed the partial pressure cross-sections $\sigma^{(1)}\left(j_{1}=0, j_{2}, E_{k i n}\right)$ for $j_{2}<8$ and in Eq. (4) used averaged values determined from the first 8 ones to take into account higher populated $j_{2}$ states. Fig. 1 shows these partial pressure broadening cross-sections for $j_{2}<8$ and illustrates the defects of the coupled states approximation (CSA); this problem is well known, see $e$. g. [35-38]. Thus, and although our quantum dynamical pressure broadening coefficients cannot be qualified as benchmarks for other methods, the comparison with selected experimental values shown in Table 1 illustrates that our calculation indeed provides good values for these coefficients. We also note that the use of Eq. (7) instead of Eq. (4) does not lead to significant changes.

The same $S$-matrix files, used to calculate the partial pressure broadening cross-sections for the $\mathrm{R}(0)$ lines, were used to calculate cross-sections for $\mathrm{Q}$ lines $\sigma^{(0)}\left(j_{1}, j_{2}, E_{k i n}\right)$. For instance, for the $\sigma^{(0)}\left(j_{1}=5, j_{2}=0, E_{k i n}\right)$ the thermal averaging was performed over $62 \mathrm{CC}$ values between 0 and $310 \mathrm{~cm}^{-1}$ and $9 \mathrm{CS}$ values between 342 and $667 \mathrm{~cm}^{-1}$ and an extrapolation was made up to about $2500 \mathrm{~cm}^{-1}$. As in [30] for $\mathrm{N}_{2}-\mathrm{N}_{2}$, one may define an ortho- and a parapressure broadening cross-sections. As already observed $[29,30]$ these two contributions are very close at 150 and

\footnotetext{
${ }^{1}$ For instance, at a kinetic energy of $250 \mathrm{~cm}^{-1}$ for $j_{2}=2$ and $j_{1}=0$, the duration of a MOLSCAT run is about 10 days with 8 cores working in parallel on two quad-core processors (Intel Xeon X5550). Recall that 2 runs are necessary to obtain the right $S$-matrix elements which contribute to the partial pressure broadening cross section of the $R(0)$ line.
} 


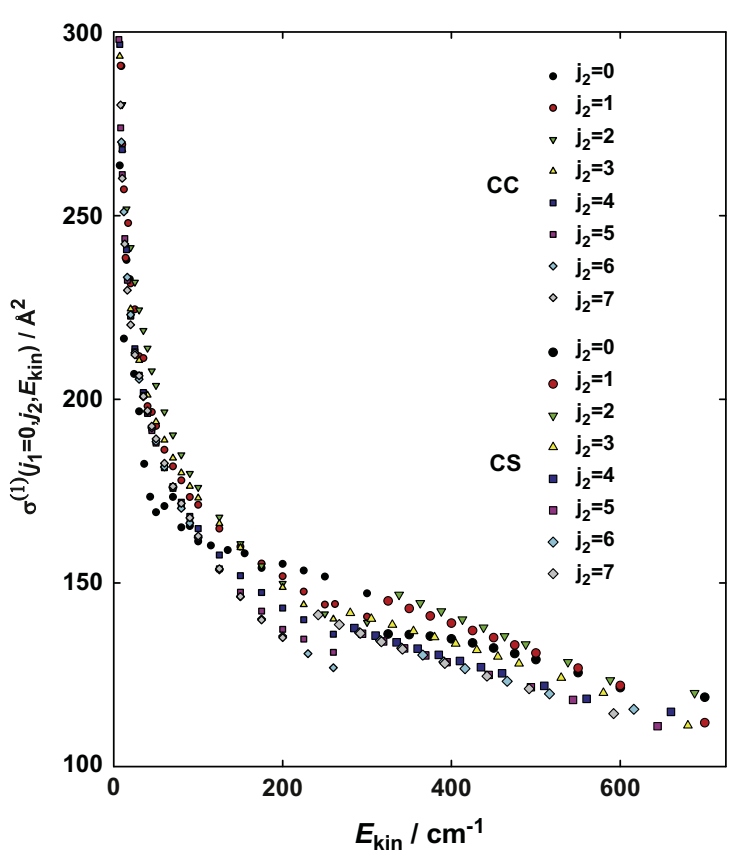

Fig. 1. Partial pressure broadening cross-sections $\sigma^{(1)}\left(j_{1}=0, j_{2}, E_{k i n}\right)$, in $\AA^{2}$, for the $\mathrm{R}(0)$ line and $j_{2}=0$ to 7 as a function of the relative kinetic energy in $\mathrm{cm}^{-1}$; CC values, small symbols, CS values, big symbols. Rotational quantum numbers $j_{1}$ and $j_{2}$ correspond to $\mathrm{C}_{2} \mathrm{H}_{2}$ and $\mathrm{N}_{2}$ molecules respectively.

$298 \mathrm{~K}$. This may be useful for subsequent authors to avoid unnecessary calculations.

\subsection{Semi-classical Robert-Bonamy method}

Dynamical calculations were performed, at the mean thermal speed associated to the room temperature, using the exact trajectory model determined only by the isotropic part of the PES and potential terms given by Eq. (3) up to $L_{1}=L_{2}=4$ (see for instance Refs. [11,18-20,22] and references therein). We have checked that higher multipolar components of the PES do not provide any significant contributions to such calculations of the pressure broadening coefficients.

The way to determine pressure broadening crosssections from the standard Robert-Bonamy formalism [12], whatever the type of transition operators, is well established. The method employed here is very similar to the one used in Ref. [18] for $\mathrm{N}_{2}-\mathrm{N}_{2}$ and therefore will not be repeated again.

Ma and coauthors [20] have refined the above mentioned formalism by considering effects from the line coupling for isotropic Raman Q-lines of a linear molecule. More specifically, by correctly evaluating the cumulant expansion of the Liouville scattering operator $\langle\widehat{S}\rangle=$ $\exp \left(-i S_{1}-S_{2}\right)$ up to the second order, they are able to take into account the line coupling for isotropic Raman Qlines whose initial and final quantum numbers are identical. The method developed in [20] cannot be straightforwardly adapted for R- or P-infrared lines because for such lines the above condition is not valid. As a result, one has to develop a new method to evaluate the matrix elements of the operator $\exp \left(i S_{1}+S_{2}\right)$. Since this subject merits a separate derivation that will not be detailed here. This new method and its application to the present system are presented somewhere else [26]. Finally, let us simply mention that in the present study, we have not considered the imaginary part of $\left(\mathrm{i} S_{1}+S_{2}\right)$. Because the interaction PES has no vibrational dependence, the $S_{1}$ term becomes zero. Given the fact that is $S_{1}$ term is the main component of Im $\left(\mathrm{i} S_{1}+S_{2}\right)$ and $\operatorname{Im}\left(S_{2}\right)$ is much smaller than $\operatorname{Re}\left(S_{2}\right),[4,8,26]$, we have decided to neglect $\operatorname{Im}\left(S_{2}\right)$ in our calculations.

\subsection{Full classical method}

The half-widths of isotropic Raman scattering and IR (electric dipole absorption) spectral lines of $\mathrm{C}_{2} \mathrm{H}_{2}$ perturbed by $\mathrm{N}_{2}$ were also calculated in the frame of the classical impact approach first proposed by Gordon [16,17]. Translational and rotational motions of $\mathrm{C}_{2} \mathrm{H}_{2}$ and $\mathrm{N}_{2}$ molecules were modeled using truly exact threedimensional classical (C3D) equations describing the collision of two rigid linear molecules (17 first-order Hamilton differential equations in body-fixed coordinates [39]). Hence, the trajectories are driven by the isotropic and the anisotropic parts of the PES. Spin statistical weights of perturbing $\mathrm{N}_{2}$ molecules were taken into account in the usual way. The initial intermolecular distance was set to a large enough figure ( $15 \AA$ ) to exclude starting interaction between molecules. Other collision parameters (initial conditions for trajectory calculations) were selected via a Monte Carlo procedure. Uniform sampling was applied to the directions of rotation and initial orientations of both molecules in three-dimensional space. The statistical error of calculated linewidths (i.e., RMS error of Monte Carlo averaging over initial conditions of collisions) was kept for different values of temperature $T$ and rotational quantum number $j$ generally at a level less than $1 \%$. The impact parameter was bracketed within the range 0-12 A. To verify that $12 \AA$ was a large enough upper limit, we conducted additional test calculations at $T=150 \mathrm{~K}$ with somewhat larger values of the maximum impact parameter, namely $b_{\max }=13 \AA$ and $15 \AA$. They gave nearly identical results to $b_{\max }=12 \AA$, confirming the fact that collisions with $b>12$ Å have little influence on line broadening in our case.

The majority of calculations were performed only at the mean thermal velocity $\bar{v}$, which greatly reduces CPU time (see Eq. (6)). Several tests were conducted confirming the validity of MThV approximation in the cases considered. At $150 \mathrm{~K}$, for instance, for both $\mathrm{R}$ and $\mathrm{Q}$ lines, relative differences between pressure broadening coefficients obtained using the MThV approximation with thermally averaged values are no more than about $4 \%$. This percentage diminishes as the temperature increases (see Fig. 2). This behavior is confirmed by quantum dynamical calculations and allows the use of Eq. (7) for the SC calculations.

As a second application of these calculations, Fig. 2 shows that pressure broadening coefficients for isotropic Raman spectral lines become closer and closer to IR electric dipole absorption lines as $j_{1}$ and $T$ increases. This fact is well known (see for instance Refs. [21,29] and references therein). 


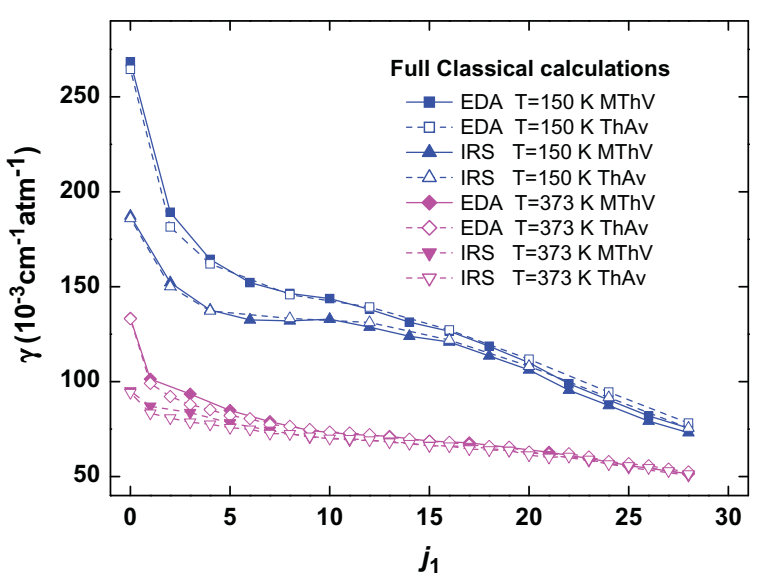

Fig. 2. Comparison of thermally averaged (ThAv) and mean thermal velocity (MThV) full classical values at $150 \mathrm{~K}$ and $373 \mathrm{~K}$ for electric dipole absorption (EDA) R-lines and isotropic Raman spectra (IRS) Q-lines and comparison of pressure broadening coefficients for such types of lines.

\section{Experimental methods}

\subsection{Experimental set-up}

In order to carry out an experimental determination of the collisional broadening coefficients of the $\mathrm{C}_{2} \mathrm{H}_{2}-\mathrm{N}_{2}$ system from isotropic Raman data, we recorded high resolution Raman spectra of mixtures of these two gases at different pressures and temperatures in the region of the Q-branch of the $v_{2}$ vibrational band of $\mathrm{C}_{2} \mathrm{H}_{2}$, at $\sim 1975 \mathrm{~cm}^{-1}$. The experimental setup used for the obtention of these spectra, built around a stimulated Raman spectrometer, has been described in detail in prior articles (see for example Ref. [29], which reports a similar collisional broadening study in $\mathrm{C}_{2} \mathrm{H}_{2}$ perturbed by $\mathrm{H}_{2}$, and references therein). While a number of improvements have been introduced in the experimental system in recent years [40], its essential characteristics remain the same, so in this article we will omit a detailed description and direct instead the interested reader to the references given above.

As in Ref. [29], the $\mathrm{Ar}^{+}$laser of the spectrometer was operated in its $529 \mathrm{~nm}$ line, and the frequency of the cw dye laser was scanned between approximately 16,935 and $16,937 \mathrm{~cm}^{-1}$. This required the use of Rhodamine $6 \mathrm{G}$ in the dye laser and Kiton Red in the pulsed dye amplifier. The frequency difference of the two laser sources thus covered the range between $\sim 1972.5$ and $1974.5 \mathrm{~cm}^{-1}$, where the $\mathrm{Q}(1)$ to $\mathrm{Q}(15)$ lines of the $\nu_{2}$ rovibrational band of $\mathrm{C}_{2} \mathrm{H}_{2}$ lie in the Raman spectrum. Q-branch lines corresponding to higher quantum numbers than $j=15$ could be observed, but their diminishing $S / N$ ratio (especially in the low-temperature spectra) and the fact that they overlap with lines from the $\nu_{2}+v_{5}-v_{5}$ hot band (clearly visible in the room-temperature spectra) made it advisable to include only lines up to $j=15$ in the analysis. The output power of the cw $\mathrm{Ar}^{+}$laser was set to $500 \mathrm{~mW}$, and the peak energy of the amplified dye pulses to $\sim 15 \mathrm{~mJ} /$ pulse at a repetition rate of $30 \mathrm{~Hz}$, corresponding to the repetition rate of the Nd:YAG laser used to pump the dye amplifier. This new laser is one of the improvements introduced in the system in recent years [40].

Diluted mixtures of $\mathrm{C}_{2} \mathrm{H}_{2}$ (3 vol\%) in $\mathrm{N}_{2}$ were used as samples. This concentration was chosen as a compromise, since it is high enough to provide good Raman signals in the range of pressures at which the experiments were conducted while, at the same time, being low enough to prevent self-broadening from having a noticeable contribution to the lineshapes in the recorded $\mathrm{C}_{2} \mathrm{H}_{2}$ spectra. The mixtures were prepared several days in advance in order to insure proper mixing.

Two sets of measurements were conducted, one of them at room temperature $(298 \mathrm{~K})$ and the other one at $150 \mathrm{~K}$. The coolable cell described in [29] was used as sample container in both cases. In each one of these sets of measurements we followed the same procedure: the cell was filled with gas mixture at a certain pressure and the Raman spectrum of the Q-branch of the $\nu_{2}$ band of $\mathrm{C}_{2} \mathrm{H}_{2}$ was recorded. Then the pressure was reduced through controlled, slow pumping and the spectrum recorded again. This procedure was repeated several times in order to obtain Raman spectra of the sample at different pressures. For the room temperature measurements these pressures were $70,60,50,40,30$ and 20 mbar, while for the ones conducted at $150 \mathrm{~K}$ they were $35,30,25,20,15$ and 10 mbar. All the measurements were carried out twice (i.e., two independent spectra were recorded at each temperature and pressure) in order to average the final results.

\subsection{Data reduction}

The spectra thus obtained were analyzed by fitting the spectral lines to Voigt profiles. The range of pressures in which the experiments were conducted might suggest that more complex profiles that include the Dicke narrowing effect should have been used, since such narrowing should already be visible in this pressure range. Indeed, we initially performed fits using Galatry and Rautian profiles. However, the $S / N$ ratio of the spectra ( $\sim 30$ for the most favorable cases) was not high enough for a clear observation, let alone quantification of the narrowing effect, and all our attempts failed to provide a reliable value of the narrowing parameter different from zero. Thus, a Voigt profile was finally deemed adequate for our analysis. Any possible underestimation of the collisional line widths derived from disregarding the narrowing effect should be well within their uncertainty intervals, since these are rather large due to the noise that prevents us from observing the narrowing in the spectra.

Another concern regarding the use of Voigt profiles is the possible presence of line mixing in the spectra. However, any line mixing effect that may affect the lineshape can be safely disregarded for two reasons. First, as shown by Bouanich et al. [13,14] in Q-branches of $\Sigma \leftarrow \Pi$ IR transitions for pressures below $0.1 \mathrm{~atm}$ line mixing is negligible, a condition fulfilled by our experiments. Secondly, since adjacent lines belong to ortho- and paraspecies, interferences are only possible between second (or higher) neighboring lines. 


\section{Intercomparisons of pressure broadening coefficients}

\subsection{Isotropic Raman lines}

Fig. 3 provides a comparison of experimental pressure broadening coefficients of Q-lines of $\mathrm{C}_{2} \mathrm{H}_{2}$ in $\mathrm{N}_{2}$ baths at 150 and $298 \mathrm{~K}$ with calculated values. Only CC/CS and FC calculations were performed at $150 \mathrm{~K}$ while the three types of calculations were performed at $298 \mathrm{~K}$. At $150 \mathrm{~K}$ the FC values are inside the large errors bars while the quantum dynamical calculations seem to underestimate the experimental values. For the latter kind of calculations the MThV values obtained at the kinetic energy of $133 \mathrm{~cm}^{-1}$, using Eq. (6), are slightly larger than the thermally CC/CS values (not shown). This may be partly due to the fact that the latter are more affected by the default of the CS approximation but also by the average integration. This difference between MThV and ThAv values diminishes at room temperature (our CC/CS values tend to be more and more CS values as the collision kinetic energies involved are larger and, generally speaking, the thermal average is less important as the kinetic energies and therefore the temperature increase, see Fig. 2). At room temperature, both the CC/CS and FC values are in quite good agreement with the experimental data. Now, considering the semi-classical values provided by the traditional RB method, we see that they are largely overestimated by about 20\%. If line coupling effects are included in the calculations (see RB method modified by Ma et al. $[20,26])$ the results are drastically improved.

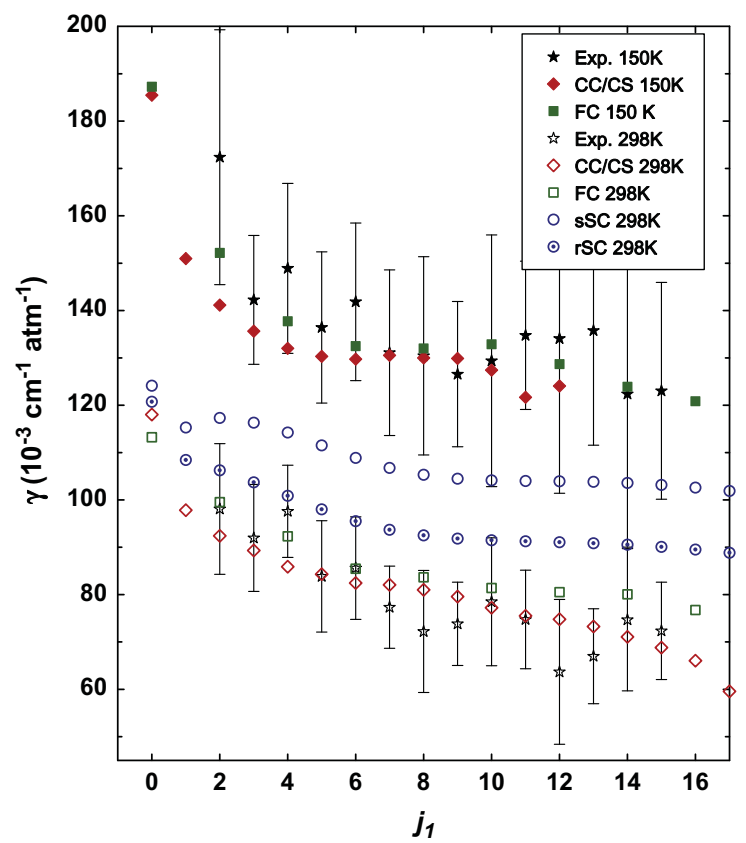

Fig. 3. Comparison of experimental (stars) pressure broadening coefficients for isotropic Raman $\mathrm{Q}\left(j_{1}\right)$ lines at 150 and $298 \mathrm{~K}$ with calculated values: full-classical (FC) method (squares), CC/CS method (diamonds), standard semi-classical method of [18] (sSC, hollow circles) and refined semi-classical method of $[20,26]$ (rSC,dotted circles).

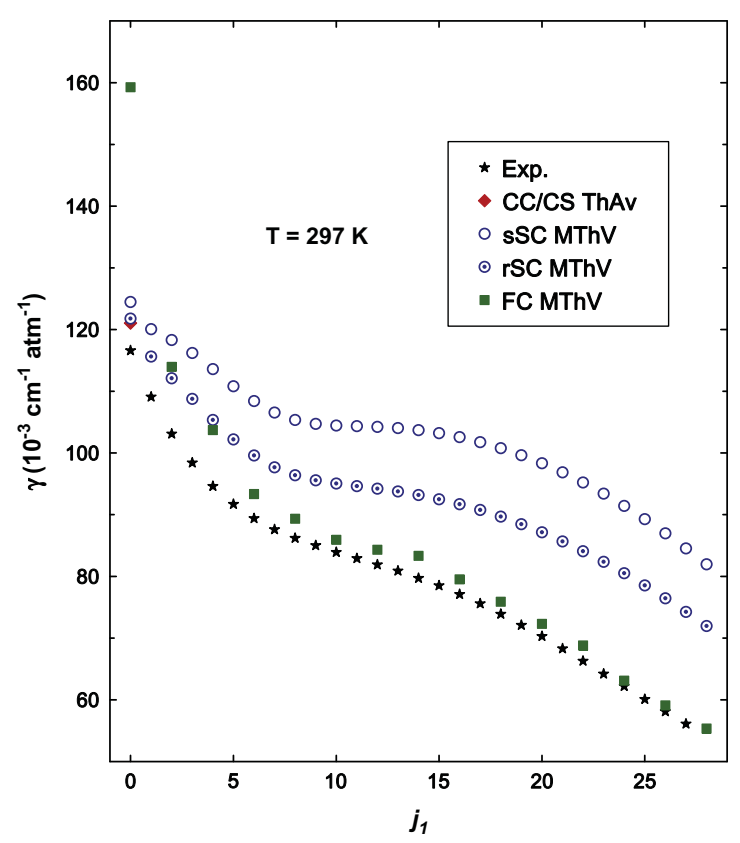

Fig. 4. Comparison between experimental (stars) room temperature pressure broadening coefficients for IR lines [1] and calculated values: FC (squares), CC/CS (diamonds), standard semi-classical [18] (sSC, hollow circles) and refined semi-classical [26] (rSC, dotted circles) methods.

\subsection{IR lines}

Experimental data at room temperature are numerous (see [9]). We have chosen to compare first our calculated values with the smoothed ones obtained from measurements in the R- and P-branches of the $\nu_{5}$ band [1] and which are reported in Fig. 4. We remind the reader that we were unable, for technical and CPU time reasons, to calculate pressure broadening coefficients for such lines except for the $\mathrm{R}(0)$ line. Here again, we note that the full classical values are in quite good agreement with the experimental values, especially for the highest $j$ values. The traditional RB semi-classical method strongly overestimates the pressure broadening coefficients whereas the new method of Ma et al. [20,26] gives the results in between the FC values (or the experimental ones) and the traditional $\mathrm{RB}$ values.

\section{Conclusions}

We have provided new experimental pressure broadening coefficients for isotropic Raman lines of acetylene in nitrogen at 150 and $298 \mathrm{~K}$. Based on these results we can claim that the quantum dynamical and full classical calculations performed on the $a b$ initio PES of Ref. [15] lead to a good agreement with these experimental data. The pressure broadening coefficients calculated with the full classical method for IR R-lines in our rigid rotor approximation as compared with various experimental data for such lines are also quite good and confirmed previous calculations performed in [9]. Unfortunately, for such lines even for a simple system like $\mathrm{C}_{2} \mathrm{H}_{2}-\mathrm{N}_{2}$, with a quite large reduced mass and small rotational constant, it 
is still too difficult to routinely handle computationally close-coupling calculations to allow a complete intercomparison. Alternative methods are therefore required.

This paper ends a series of our articles [21-23] devoted to an intercomparison of quantum dynamical, full classical and traditional semi-classical (Robert and Bonamy) formalisms. The standard Robert-Bonamy method [12] is unable to provide accurate pressure broadening coefficients without adjusting the PES. It is well known [18$23,26]$ that the standard Robert-Bonamy formalism suffers from several weaknesses. Among them, there are two particularly severe ones. The first is to treat the translational motion and the internal motion separately. More specifically, the exchanges of their translational kinetic and internal rotational energies are not considered during a collision process and trajectories are assumed to be independent of anisotropic potential models. The second one is to neglect effects from the line coupling in calculations. In order to improve the semi-classical method such that it can serve as a well-qualified alternative to the quantum dynamical formalisms for considering any kind of interacting molecular pairs, one must directly address these weaknesses. As shown in the previous study for selfbroadening coefficients of $\mathrm{N}_{2}$ lines [20] and in the currently going study for the $\mathrm{C}_{2} \mathrm{H}_{2}$ molecule [26] whose rotational constant is even smaller than that of $\mathrm{N}_{2}$, taking into account the line coupling significantly improves results derived from the traditional semi-classical calculations. This implies that the refinement effort goes in the right direction.

\section{Acknowledgments}

D.B. and R.Z.M. acknowledge the financial support of the Ministry of Economy and Competitiveness through Research Grant FIS2012-38175. Q.M. acknowledges the financial support from NSF under Grant 1228861. D.B., R.Z.M and F.T. acknowledge financial support from the European GDRI HiRESMIR (high resolution microwave, infrared and Raman molecular spectroscopy).

\section{Appendix A}

Since CC or CS computations of pressure broadening cross-sections are very scarce [21-23,29,30,32,41-46] for the case of two linear molecules, this appendix recalls a few formulae. Following Ben-Reuven [27] and later on Coombe et al. [31], Green [32] and Monchick et al. [33], the internal motion of the active molecule is disentangled from the bath. The rotational motion of the perturbing molecule "2" is first coupled with the relative kinetic angular momentum $\ell$ to form $\bar{j}$ and then $j_{1}$ is coupled to it to provide the total angular momentum $J\left(\vec{J}=\vec{j}_{1}+\overrightarrow{\vec{j}}\right)$. This choice is more natural when one is interested to relaxation phenomena tied to the active molecule. In this coupling scheme the $\mathrm{CC}$ generalized pressure broadening cross-section is given by

$$
\begin{aligned}
\sigma^{(n)} & \left(j_{a} j_{b} j_{2}^{\prime}, j_{a} j_{b} j_{2} ; E_{k i n}\right) \\
= & \left(\frac{\pi}{k^{2}}\right) \frac{1}{\left(2 j_{2}+1\right)} \sum_{J_{a} J_{b} \bar{j} \bar{j}^{\prime} \ell \ell^{\prime}}\left[J_{a}\right]\left[J_{b}\right](-1)^{\bar{j}-\bar{j}^{\prime}} \\
& \times\left\{\begin{array}{ccc}
j_{a} & n & j_{b} \\
J_{b} & \bar{j}^{\prime} & J_{a}
\end{array}\right\}\left\{\begin{array}{lll}
j_{a} & n & j_{b} \\
J_{b} & \bar{j} & J_{a}
\end{array}\right\} \times\left[\delta_{j_{2} j_{2}^{\prime}} \delta_{\bar{j} \bar{j}^{\prime}} \delta_{\ell \ell^{\prime}}\right. \\
& -<j_{a}\left(j_{2}^{\prime} \ell^{\prime}\right) \bar{j}^{\prime} \mid S^{J_{a}}\left(E_{k i n}+E_{j_{a}}+E_{j_{2}}\right)\left(j_{a}\left(j_{2} \ell\right) \bar{j}>\right. \\
& <j_{b}\left(j_{2}^{\prime} \ell^{\prime}\right) \bar{j}^{\prime} \mid S^{J_{b}}\left(E_{k i n}+E_{j_{b}}+E_{j_{2}}\right)\left(j_{b}\left(j_{2} \ell\right) \bar{j}>*\right]
\end{aligned}
$$

where $j_{a}, j_{b}$ characterize a spectroscopic transition with rank $n$ of the radiation. $k$ is the wavevector of the relative motion: $E_{\text {kin }}=2 \mu k^{2}$.

To be compared with experimental values, these crosssections must be summed over final perturber levels $j_{2}^{\prime}$ and averaged over initial bath conditions:

$\sigma^{(n)}\left(j_{1} ; T\right)=\sum_{j_{2}} \rho_{j_{2}} \sigma^{(n)}\left(j_{1}, j_{2} ; T\right)$,

where we have used a shortcut notation $\left(j_{1}=j_{a}, j_{b}=j_{a}+1\right.$ for R lines and $j_{b}=j_{a}$ for isotropic Raman lines) and

$\sigma^{(n)}\left(j_{1}, j_{2} ; T\right)=\sum_{j_{2}^{\prime}} \sigma^{(n)}\left(j_{1}, j_{2}, j_{2}^{\prime} ; T\right)$.

The MOLSCAT code [28] does not provide generalized cross-sections (Eq. (A1)) except the full diagonal term $\left(j_{2}=j_{2}^{\prime}\right)$. Moreover, the $S$-matrix elements are given in the traditional scattering scheme where $j_{1}$ and $j_{2}$ are first coupled to give $j_{12}$. Therefore we have written a postprocessor code starting with $S^{J}\left(\left(j_{1} j_{2}\right) j_{12} \ell \mid\left(j_{1}^{\prime} j_{2}^{\prime}\right) j_{12}^{\prime} \ell\right)$ as given by the MOLSCAT code to obtain $S^{J}\left(j_{1}\left(j_{2} \ell\right) \bar{j}\right)$ matrix elements [32].

Such cross-sections calculations are not at all implemented in the MOLSCAT code for diatomic-diatomic (linear-linear) molecules within the CSA. To this end we have used $S$-matrix elements, $S^{\ell m_{\ell}}\left(\left(j_{1} j_{2}\right) j_{12} \mid\left(j_{1}^{\prime} j_{2}^{\prime}\right) j_{12}^{\prime}\right)$, as given by the MOLSCAT code and coded Eq. (96) of Goldflam and Kouri [34] in another post-processor program.

\section{References}

[1] Bouanich JP, Lambot D, Blanquet $\mathrm{G}$, Walrand J. $\mathrm{N}_{2}$ - and $\mathrm{O}_{2}$-broadening coefficients of $\mathrm{C}_{2} \mathrm{H}_{2}$ IR lines. J Mol Spectrosc 1990;140: 195-213.

[2] Pine AS. Self-, $\mathrm{N}_{2}$ - and Ar-broadening and line mixing in $\mathrm{HCN}$ and $\mathrm{C}_{2} \mathrm{H}_{2}$. J Quant Spectrosc Radiat Transf 1993;50:149-66.

[3] Bouanich JP, Blanquet G, Populaire JC, Walrand J. Nitrogen broadening of acetylene lines in the $\nu_{5}$ band at low temperature. J Mol Spectrosc 1998;190:7-14.

[4] Babay A, Ibrahimi M, Lemaire V, Lemoine B, Rohart F, Bouanich JP. Line frequency shifting in the $\nu_{5}$ band of $\mathrm{C}_{2} \mathrm{H}_{2}$. J Quant Spectrosc Radiat Transf 1998;59:195-202.

[5] Arteaga SW, Bejger CM, Gerecke JL, Hardwick JL, Martin ZT, Mayo J, et al. Line broadening and shift coefficients of acetylene at $1550 \mathrm{~nm}$. J Mol Spectrosc 2007;243:253-66.

[6] Dhyne M, Fissiaux L, Populaire JC, Lepère M. Temperature dependence of the $\mathrm{N}_{2}$-broadening coefficients of acetylene. J Quant Spectrosc Radiat Transf 2009;110:358-66.

[7] Dhyne M, Joubert P, Populaire JC, Lepère M. Collisional broadening and shift coefficients of lines in the $\nu_{4}+\nu_{5}$ band of ${ }^{12} \mathrm{C}_{2} \mathrm{H}_{2}$ diluted in $\mathrm{N}_{2}$ from low to room temperatures. J Quant Spectrosc Radiat Transf 2010;111:973-89.

[8] Rozario H, Garber J, Povey C, Hurtmans D, Buldyreva J, Predoi-Cross A. Experimental and theoretical study of $\mathrm{N}_{2}$-broadened acetylene line parameters in the $\nu_{1}+v_{3}$ band over a range of temperatures. Mol Phys 2012;110:2645-63.

[9] Povey C, Guillorel-Obregon M, Predoi-Cross A, Ivanov SV, Buzykin OG, Thibault F. Low pressure line shape study of nitrogen perturbed 
acetylene transitions in the $v_{1}+v_{3}$ band over a range of temperatures. Can J Phys 2013;91:896-905.

[10] Buldyreva J, Nguyen L. On the role of trajectory modelling in the $\mathrm{C}_{2} \mathrm{H}_{2}$ infrared line-broadening computation. Mol Phys 2004;102: 1523-35.

[11] Buldyreva J, Bonamy J, Weikl M, Beyrau F, Seeger T, Leipertz A, et al. Linewidth modelling of $\mathrm{C}_{2} \mathrm{H}_{2}-\mathrm{N}_{2}$ mixtures tested by rotational CARS measurements. J Raman Spectrosc 2006;37:647-54.

[12] Robert D, Bonamy J. Short range force effects in semiclassical molecular line broadening calculations. J Phys (Paris) 1979;40: 923-43.

[13] Bouanich JP, Blanquet G, Walrand J. Line-mixing effects in He- and $\mathrm{N}_{2}$-broadened $\Sigma \leftarrow \Pi$ Infrared Q Branches of $\mathrm{C}_{2} \mathrm{H}_{2}$. J Mol Spectrosc 2000;203:41-8.

[14] Blanquet G, Walrand J, Bouanich JP. Line-mixing effects in He- and $\mathrm{N}_{2}$-broadened $\mathrm{Q}$ branches of $\mathrm{C}_{2} \mathrm{H}_{2}$ at low temperatures. J Mol Spectrosc 2001;210:1-7.

[15] Thibault F, Vieuxmaire O, Sizun T, Bussery-Honvault B. An ab initio potential energy surface for the $\mathrm{C}_{2} \mathrm{H}_{2}-\mathrm{N}_{2}$ system. Mol Phys 2012;110:2761-71.

[16] Gordon RG. Theory of the width and shift of molecular spectral lines in gases. J Chem Phys 1966;44:3083-9.

[17] Gordon RG. Semiclassical theory of spectra and relaxation in molecular gases. J Chem Phys 1966;45:1649-55.

[18] Ma Q Tipping RH, Boulet C. Irreducible correlation functions of the S matrix in the coordinate representation: application in calculating Lorentzian half-widths and shifts. J Chem Phys 2006;124 0141109-1-14.

[19] Ma Q Tipping RH, Boulet C. Modification of the Robert-Bonamy formalism in calculating Lorentzian half-widths and shifts. J Quant Spectrosc Radiat Transf 2007; 103:588-96.

[20] Ma Q Boulet C, Tipping RH. Refinement of the Robert-Bonamy formalism: Considering effects from the line coupling. J Chem Phys 2013;139:034305-1-16.

[21] Thibault F, Ivanov SV, Buzykin OG, Gomez L, Dhyne M, Joubert P, et al. Comparison of classical, semiclassical and quantum methods in hydrogen broadening of acetylene lines. J Quant Spectrosc Radiat Transf 2011;112:1429-37.

[22] Gomez L, Ivanov SV, Buzykin OG, Thibault F. Comparison of quantum, semiclassical and classical methods in hydrogen broadening of nitrogen lines. J Quant Spectrosc Radiat Transf 2011;112: 1942-9.

[23] Thibault F, Gomez L, Ivanov SV, Buzykin OG, Boulet C. Comparison of quantum, semiclassical and classical methods in the calculation of nitrogen self-broadened linewidths. J Quant Spectrosc Radiat Transf 2012;113:1887-97.

[24] Thibault F, Boulet C, Ma Q. Line coupling effects in the isotropic Raman spectra of $\mathrm{N}_{2}$ : a quantum calculation at room temperature. J Chem Phys 2014;140:044303-1-6.

[25] Boulet C, Ma Q Thibault F. Line interference effects using a refined Robert-Bonamy formalism: the test case of the isotropic Raman spectra of autoperturbed $N_{2}$. J Chem Phys 2014;140:084310-1-8.

[26] Ma Q Boulet C, Tipping RH. Two dimensional symmetric correlation functions of the $\hat{S}$ operator and two dimensional Fourier transforms: considering the line coupling for $\mathrm{P}$ and $\mathrm{R}$ lines of a linear molecule. J Chem Phys 2014;140:104304-1-13.

[27] Ben-Reuven A. Symmetry considerations in pressure-broadening theory. Phys Rev 1966;141:34-40;

Ben-Reuven A. Impact broadening of microwave spectra. Phys Rev 1966; 145:7-22;

Ben-Reuven A. Resonance broadening of spectral lines. Phys Rev A 1971;4:2115-20.

[28] Hutson JM, Green S. MOLSCAT version 14, Collaborative Computational Project 6 of the UK Science and Engineering Research Council, Daresbury Laboratory, UK, 1995. George C. McBane, "PMP Molscat", a parallel version of Molscat version 14 available at http://faculty. gvsu.edu/mcbaneg/pmpmolscat, Grand Valley State University (2005).

[29] Thibault F, Corretja B, Viel A, Bermejo D, Martinez RZ, BusseryHonvault B. Linewidths of $\mathrm{C}_{2} \mathrm{H}_{2}$ perturbed by $\mathrm{H}_{2}$ : experiments and calculations from an $a b$ initio potential. Phys Chem Chem Phys 2008;10:5419-28.

[30] Thibault F, Martinez RZ, Bermejo D, Gomez L. Collisional line widths of autoperturbed $\mathrm{N}_{2}$ : measurements and quantum calculations. J Quant Spectrosc Radiat Transf 2011;112:2542-51.

[31] Coombe DA, Snider RF. Sanctuary BC. Definitions and properties of generalized collision cross sections. J Chem Phys 1975;63:3015-30.

[32] Green S. Rotational excitation in collisions between two rigid rotors: alternate angular momentum coupling and pressure broadening of $\mathrm{HCl}$ by $\mathrm{H}_{2}$. Chem Phys Lett 1977;47:119-22.

[33] Monchick L, Hunter LW. Diatomic-diatomic molecular collision integrals for pressure broadening and Dicke narrowing: a generalization of Hess's theory. J Chem Phys 1986;85:713-8.

[34] Goldflam R, Kouri DJ. On angular momentum decoupling approximations and factorization in diatom-diatom scattering. J Chem Phys 1979;70:5076-91.

[35] Kouri DJ. In: Bernstein RB, editor. Atom-molecule collision theory: a guide for the experimentalist. New York: Plenum; 1979. p. 301-58.

[36] Roche CF, Dickinson AS, Hutson JM. A failing of coupled-states calculations for inelastic and pressure-broadening cross sections: calculations on $\mathrm{CO}_{2}-\mathrm{Ar}$. J Chem Phys 1999;111:5824-8.

[37] Thibault F, Martinez RZ, Domenech JL, Bermejo D, Bouanich JP. Raman and infrared linewidths of CO in Ar. J Chem Phys 2002;117: 2523-31.

[38] Martinez RZ, Domenech JL, Bermejo D, Thibault F, Bouanich JP, Boulet C. Close coupling calculations for rotational relaxation of $\mathrm{CO}$ in argon. Accuracy of energy corrected sudden scaling procedures and comparison with experimental data. J Chem Phys 2003;119: 10563-74.

[39] Ivanov SV, Buzykin OG. Classical calculation of self-broadening in $\mathrm{N}_{2}$ Raman spectra. Mol Phys 2008;106:1291-302.

[40] Di Lonardo G, Fusina L, Baldan A, Martínez RZ, Bermejo D. High resolution infrared and Raman spectroscopy of $\nu_{2}$ and associated combination and hot bands of ${ }^{13} \mathrm{C}^{12} \mathrm{CD}_{2}$. Mol Phys 2011;109: 2533-42.

[41] Huo WM, Green S. Quantum calculations for rotational energy transfer in nitrogen molecule collisions. J Chem Phys 1996;104: 7572-89.

[42] Mengel M, Flatin DC, De Lucia FC. Theoretical and experimental investigation of pressure broadening and line shift of carbon monoxide in collision with hydrogen between 8 and $600 \mathrm{~K}$. J Chem Phys 2000;112:4069-75.

[43] Bruet X, Bonamy J, Dubernet-Tuckey ML. Comparison between semiclassical and quantum calculations of line broadening and shifting coefficients for the system $\mathrm{H}_{2}-\mathrm{H}_{2}$. Chem Phys 2000;254: 297-307.

[44] Gomez L, Martinez RZ, Bermejo D, Thibault F, Joubert P, BusseryHonvault B, et al. Q-Branch linewidths of $\mathrm{N}_{2}$ perturbed by $\mathrm{H}_{2}$ : experiments and quantum calculations from an $a b$ initio potential. J Chem Phys 2007;126:204302-1-8.

[45] Thibault F, Fuller EP, Grabow KA, Hardwick JL, Marcus CI, Marston D, et al. Experimental line broadening and line shift coefficients of the acetylene $\nu_{1}+v_{3}$ band pressurized by hydrogen and deuterium and comparison with calculations. J Mol Spectrosc 2009;256:17-27.

[46] Thibault F, Cappelletti D, Pirani F, Bartolomei M. A bond-bond description of the intermolecular interaction energy: the case of the weakly bound acetylene-hydrogen complex. J Phys Chem A 2009;113:14867-74. 